\title{
HPLC Retention Behaviors of Poly-aromatic-hydrocarbones on Cu(II)- octabromotetrakis(4-carboxyphenyl)porphine Derivatives-Immobilized Aminopropyl Silica Gels in Polar and Non-Polar Eluents
}

\author{
Masaki Mifune, ${ }^{* a}$, Kenji Kawata,$^{b}$ Kurumi Tanaka, ${ }^{b}$ Youji Kitamura, ${ }^{a}$ Ikuko TsuKamoto, ${ }^{c}$ \\ Madoka SaIto, ${ }^{b, c}$ Jun HaginaKa, ${ }^{d}$ and Yutaka SaIto ${ }^{a}$ \\ ${ }^{a}$ Department of Pharmaceutical Sciences, Graduate School of Medicine and Dentistry and Pharmaceutical Sciences, \\ Okayama University; ${ }^{b}$ Department of Pharmaceutical Chemistry, Graduate School of Natural Science and Technology, \\ Okayama University; Tsushima-Naka, Okayama 700-8530, Japan: ${ }^{c}$ Faculty of Medicine, Kagawa University; Ikenobe, \\ Miki-cho, Kagawa 761-0793, Japan: and ${ }^{d}$ Faculty of Pharmaceutical Sciences, Mukogawa Women's University; Koshien \\ Kubancho, Nishinomiya 653-8179, Japan.
}

Received September 23, 2005; accepted October 26, 2005; published online October 31, 2005

As one of approaches of developing novel HPLC stationary phases, we prepared Cu-octabromotetrakis(4carboxyphenyl)porphine derivative-immobilized silica gels $\left(\mathrm{Cu}-\mathrm{OBTCPP}{ }_{\mathrm{D}}\right)$, and evaluated the availability of the resultant $\mathrm{Cu}-\mathrm{OBTCPP}$ as a stationary phase for separation of poly-aromatic-hydrocarbons (PAHs) and their related compounds. A Cu-OBTCPP ${ }_{D}$ column was revealed to have an ability to separate simple PAHs and be useful as a stationary phase in both polar and non-polar eluents. The retention property of the $\mathrm{Cu}-\mathrm{OBTCPP}_{\mathrm{D}} \operatorname{column}$ was evaluated in various comparative experiments using commercially available columns. In comparison with 2(1-pyrenyl)ethyl dimetylsilyl silica gel column (PYE column) regarding the retention behavior for PAHs etc., the Cu-OBTCPP ${ }_{D}$ column showed stronger interactions involving $\pi$ electron in non-polar eluent than PYE column. In comparison with a pentabromobenzyloxy propylsilyl silica gel column (PBB column) regarding the influence of bromination, the Cu-OBTCPP ${ }_{D}$ column was affected differently from the PBB column. In comparison with nitrophenylethyl silica gel column (NPE column) regarding the retention behavior for compounds having a dipole in a non-polar eluent, the $\mathrm{Cu}-\mathrm{OBTCPP}$ column showed electrostatic interactions such as dipole-dipole interaction equivalent to or larger than the NPE column.

Key words novel stationary phase; $\pi-\pi$ electron interaction; $\pi-d$ interaction; dispersion force; dipole-dipole interaction

HPLC is conventionally used for separation and/or analysis of substances such as medicines, mutagens, environmental pollutants, and the like, which are broadly present in the circumstances. $^{1-6)}$ For such a purpose, an octadecylsilyl silica gel column has still been used as a stationary phase for a preparative purpose. $^{7-9)}$ On the other hand, it has been attempted to separate and/or analyze these substances making use of interactions involving $\pi$ electron because the substances to be separated or analyzed include poly-aromatichydrocarbons (PAHs) and heterocyclic compounds abundantly. As one of approaches, stationary phases having an ability to interact with PAHs through $\pi$ electron, that is, stationary phases composed of a support carrying a compound with $\pi$ electron have been developed. ${ }^{10)}$ Currently, there are commercially available columns in this form, such as a PYE column whose stationary phase comprises a 2-(1pyrenyl)ethyl group. The applicability to the separation of PAHs and the like has already been reported..$^{6,10,11)}$ Metal porphyrin analogs such as metal porphyrin or metal phthalocyanine belong to macrocyclic compounds having a wide spread of $\pi$ electron cloud. Cu-porphyrin-immobilized silica gels have been reported to be useful as an HPLC stationary phase for separation of compounds having $\pi$ electron such as PAHs. ${ }^{12-15)}$ In addition, columns having ability to exert both the $\pi$ electronic- and electrostatic-interactions such as a pentabromobenzyloxy propylsilyl silica gel column (PBB column $)^{16)}$ and a nitrophenylethylsilyl silica gel column (NPE column) ${ }^{17)}$ have been developed and made commercially available to date.

In view of the above, we reached the conception that a novel stationary phase for PAHs and its analogous compounds may be obtained by brominating $\mathrm{Cu}$-tetrakis(4-carboxyphenyl)porphine (Cu-TCPP, see Fig. 1) ${ }^{18)}$ known to show $\pi-\pi$ electron interactions with PAHs, because bromination would destroy the planarity of porphyrin ring and change the polarity, thereby providing $\mathrm{Cu}$-TCPP with an ability to exert additional interaction(s), as is the case with PBB column. In the present research, we prepared silica gels immobilizing $\mathrm{Cu}$-octabromotetrakis(4-carboxyphenyl)porphine derivatives to give a prospective stationary phase $(\mathrm{Cu}-$ $\mathrm{OBTCPP}_{\mathrm{D}}$ ), and elucidated their retention property from various aspects. The research revealed that $\mathrm{Cu}-\mathrm{OBTCPP}_{\mathrm{D}}$ has excellent function based on interactions involving $\pi$ electron, electrostatic force, and the like, as explained in more detail below.

\section{Experimental}

Chemicals and Reagents Tetrakis-(4-carboxyphenyl)porphine $\left(\mathrm{H}_{2-}\right.$ TCPP) was obtained from Tokyo Kasei, Japan. As a support, Develosil $\mathrm{NH}_{2}-$ 5 (hereinafter, referred to as "Dev"), which is spherical aminopropyl-silica gels (particle size, $5 \mu \mathrm{m}$; specific surface area, $250 \mathrm{~m}^{2} / \mathrm{g}$; average pore size, $12 \mathrm{~nm}$, amino-group; $98 \mu \mathrm{mol} / \mathrm{g}$ ) was purchased from Nomura Kagaku, Japan. As eluents, purified water by Labo IonPure-12 (Millipore, U.S.A.), methanol and $n$-hexane for HPLC (Nacalai Tesque, Japan) were mainly used. As a solvent for packing $\mathrm{Cu}-\mathrm{OBTCPP}_{\mathrm{D}}$ and Dev, slurry solvent A Conc. (Chemco Scientific, Japan) was used. The commercially available PYE, PBB and NPE columns (particle size; $5 \mu \mathrm{m}$ : column size; $4.6 \mathrm{~mm}$ i.d. $\times 150 \mathrm{~mm}$ ), that were purchased from Nacalai Tesque, Japan, were used for comparison to elucidate the retention property and underlying separation mechanism of the $\mathrm{Cu}-\mathrm{OBTCPP}_{\mathrm{D}}$ column. Other reagents were of analytical or reagent grade.

Preparation of $\mathrm{Cu}-\mathrm{OBBTCP}_{\mathrm{D}}$ Column $\mathrm{Cu}$-TCPP was synthesized according to the method described in a literature. ${ }^{19)} \mathrm{H}_{2}$-TCPP $(50 \mathrm{mg})$ was dis- 
solved in $0.2 \mathrm{~mol} / 1$ aqueous sodium acetate solution and refluxed for $1 \mathrm{~h}$. After adding copper acetate $(20 \mathrm{mg})$, the mixture was refluxed for $30 \mathrm{~min}$ and allowed to cool. Conc. $\mathrm{HCl}$ was added to the reaction solution to precipitate $\mathrm{Cu}-\mathrm{TCPP}$. The resultant precipitates were collected by filtration, washed with water and dried over $\mathrm{P}_{4} \mathrm{O}_{10}$ under reduced pressure to yield $\mathrm{Cu}$-TCPP.

Cu-OBTCPP (see Fig. 1) was prepared as follows. Cu-TCPP $(40 \mathrm{mg})$ was dissolved in dimethyl formamide $(30 \mathrm{ml})$, and thereto is added bromosuccinimide $(144 \mathrm{mg})$. The mixture was heated at $70^{\circ} \mathrm{C}$ for $1 \mathrm{~h}$ and allowed to cool. After adding water, the resultant precipitates were collected by filtration and dried over $\mathrm{P}_{4} \mathrm{O}_{10}$ under reduced pressure to yield Cu-OBTCPP. The absorption spectrum of the obtained Cu-OBTCPP completely agreed with that in the literature. ${ }^{20)}$

Acid chloride of $\mathrm{Cu}-\mathrm{OBTCPP}$ (Cu-OBTCPPCl, see Fig. 1) was synthesized from $\mathrm{Cu}-\mathrm{OBTCPP}$ as described by Iwado et al. ${ }^{21)}$ The resultant $\mathrm{Cu}-$ OBTCPPCl $(c a .4 \mathrm{mg})$ was dissolved in dry dioxane $(10 \mathrm{ml})$. After adding Dev ( $c a .1 .5 \mathrm{~g}$ ), the mixture was refluxed for $2 \mathrm{~h}$ and allowed to cool. $\mathrm{Cu}-$ OBTCPP $_{\mathrm{D}}$ was then separated by filtration, suspended in methanol $(100 \mathrm{ml})$, and refluxed for $1 \mathrm{~h}$. Cu-OBTCPP ${ }_{D}$ was filtered off and washed with methanol $(100 \mathrm{ml})$, dried thoroughly in vacuo over $\mathrm{P}_{4} \mathrm{O}_{10}$. The Cu-OBTCPP column was prepared by packing the so obtained $\mathrm{Cu}-\mathrm{OBTCPP}{ }_{\mathrm{D}}$ into a stainless-steel column $(4.6 \mathrm{~mm}$ i.d. $\times 150 \mathrm{~mm})$ by a conventional slurry packing method. Throughout the processes, the filtrate and the like were clear and colorless, and the absorption spectra thereof were free from characteristic absorption band(s) of Cu-OBTCPP and/or Cu-OBTCPPCl. From these evidences, it was concluded that $\mathrm{Cu}$-OBTCPP was completely immobilized on the silica gels. The amount of $\mathrm{Cu}-\mathrm{OBTCPPCl}$ immobilized was estimated from the absorption spectrum of the initial $\mathrm{Cu}-\mathrm{OBTCPPCl}$ solution in dry dioxane. $\mathrm{Cu}-\mathrm{OBTCPP}_{\mathrm{D}}$ columns prepared are stable for at least 1 year.

Apparatus The HPLC system was mainly constructed with a Shimadzu LC-10AT pump, a Shimadzu SPD-10A detector, a Shimadzu Chromatopac C-R6A recorder and a Rheodyne model 7161 sample injector (Rheodyne, U.S.A.). Typical HPLC conditions are as follows: column temperature, ambient; detection, $264 \mathrm{~nm}$ in principle; eluent, $80 \%$ methanol or $100 \% \mathrm{n}$ hexane at a flow rate of $0.5 \mathrm{ml} / \mathrm{min}$.

Samples Sample compounds include 1- to 4-membered PAHs shown in Fig. 2 (Kanto Kagaku, Tokyo Kasei, Nacalai Tesque and Wako Pure Chemical Ind., Japan), fluorobenzene, chlorobenzene, iodobenzene (Nacalai Tesque), bromobenzene, benzonitrile (Ishidzu Pharm. Co. Ltd.) and nitrobenzene (Katayama Chem. Ind.). Sample solutions were $250 \mu \mathrm{g} / \mathrm{ml}$ benzene and its derivatives, and 5-20 $\mu \mathrm{g} / \mathrm{ml}$ PAHs solutions in $80 \%$ methanol or

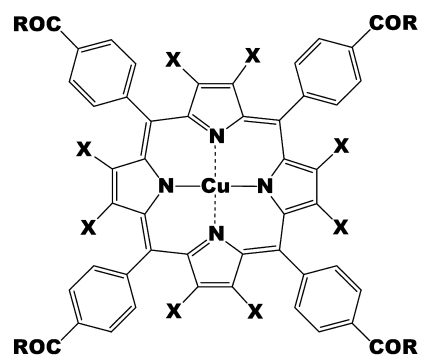

$\mathrm{X}=\mathrm{H}, \mathrm{R}=\mathrm{OH}$ : Cu-Tetrakis(4-carboxyphenyl)porphine (Cu-TCPP)

$\mathrm{X}=\mathrm{Br}, \mathrm{R}=\mathrm{OH}$ : Cu-Octabromotetrakis(4-carboxyphenyl)porphine (Cu-OBTCPP)

$\mathrm{X}=\mathrm{Br}, \mathrm{R}=\mathrm{Cl}$ : Acid chloride of Cu-OBTCPP (Cu-OBTCPPCI)

Fig. 1. Structures of Cu-Porphines

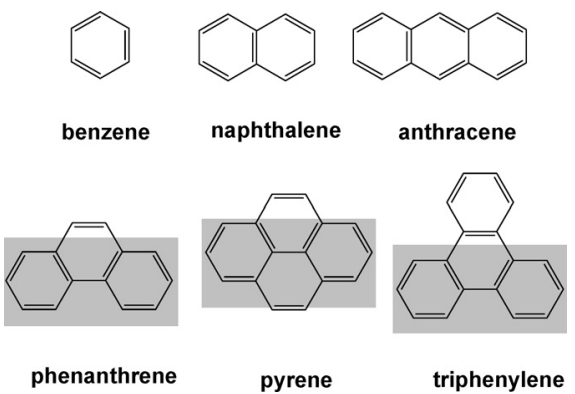

Fig. 2. The Structure of Samples
$100 \% n$-hexane

\section{Results and Discussion}

$\mathrm{Cu}$-OBTCPP differs from $\mathrm{Cu}$-TCPP in that it takes a nonplanar structure due to the repulsion between bromines and phenyl rings. ${ }^{22,23)}$ Accordingly, Cu-OBTCPP immobilized on aminopropyl-silica gels (Dev) was thought to be reduced or deprived of the ability to interact with PAHs through $\pi-\pi$ electron interaction or the like. We, therefore, prepared $\mathrm{Cu}$ OBTCPP-immobilized silica gels $\left(\mathrm{Cu}-\mathrm{OBTCPP}_{\mathrm{D}}\right)$ and evaluated whether or not it shows any interactions such as $\pi-\pi$ electron interaction and has potential usefulness as a stationary phase. Furthermore, to elucidate the retention property of PAHs on the Cu-OBTCPP ${ }_{D}$ column, comparative experiments were conducted using commercially available columns of which retention property is known.

Effect of Immobilized Amount of Cu-OBTCPP ${ }_{D}$ on Retention Factor for PAHs The retention factor $(k)$ of PAHs on $\mathrm{Cu}-\mathrm{OBTCPP}_{\mathrm{D}}$ column must vary depending on the amount immobilized on silica gels subject that Cu-OBTCPP on Dev interacts with PAHs through $\pi-\pi$ electron interaction, and the like. We therefore prepared the $\mathrm{Cu}-\mathrm{OBTCPP}_{\mathrm{D}} \mathrm{S}$ each being different in the amount of immobilized $\mathrm{Cu}$ OBTCPP within the range of 3 to $15 \mu \mathrm{mol} / \mathrm{g}$. The resultant $\mathrm{Cu}-\mathrm{OBTCPP} \mathrm{D}_{\mathrm{D}}$ was packed in a column and $k$ values of PAHs in either polar or non-polar eluent was calculated. Figures 3 and 4 show the results obtained using simple 1- to 4-membered PAHs. As can be seen from Figs. 3 and 4, PAHs are re-

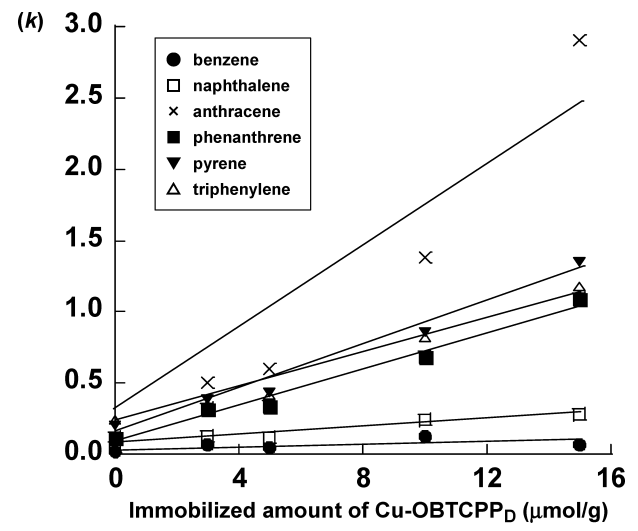

Fig. 3. Effect of Immobilized Amount of Cu-OBTCPP ${ }_{D}$ Columns on Retention Factor $(k)$ in $80 \%$ Methanol

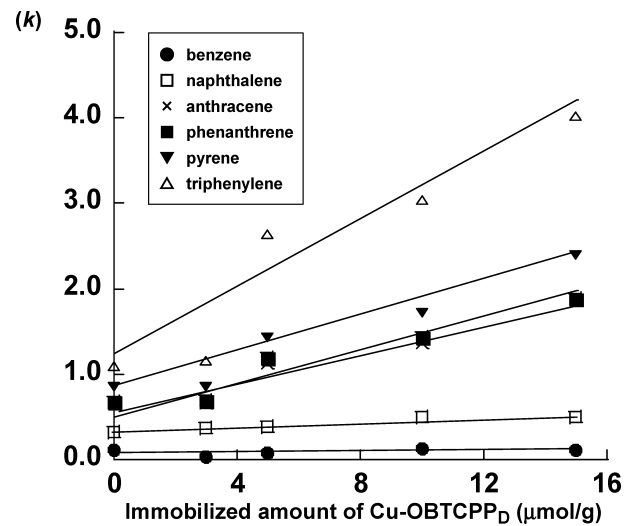

Fig. 4. Effect of Immobilized Amount of Cu-OBTCPP ${ }_{D}$ Columns on Retention Factor $(k)$ in $100 \% n$-Hexane 

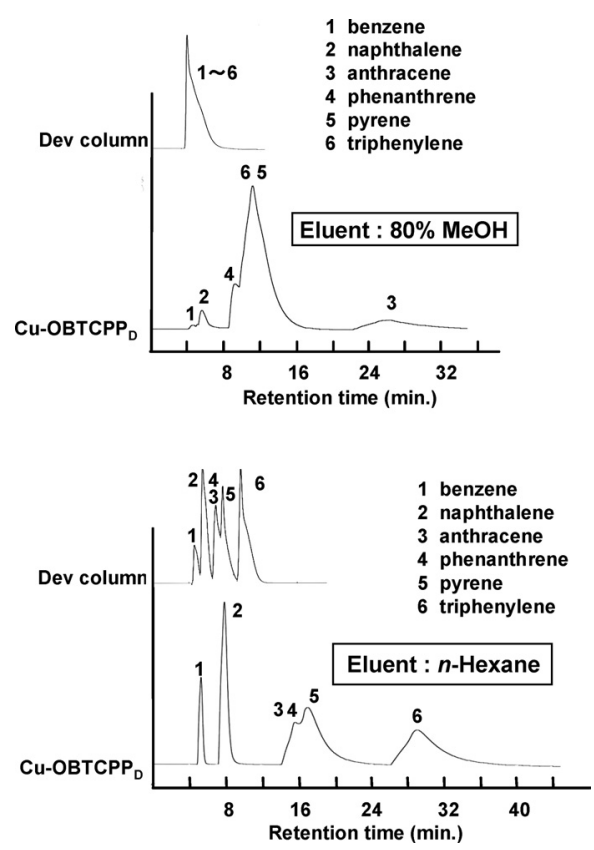

Fig. 5. The Chromatogram of Samples on Dev and Cu-OBTCPP Columns

tained slightly even by Dev alone in both polar- and nonpolar-eluents; but $k$ values of PAHs became larger as the amount of immobilized Cu-OBTCPP increased. This means that $\mathrm{Cu}-\mathrm{OBTCPP}_{\mathrm{D}}$ actually interacts with PAHs and that $\mathrm{Cu}-$ OBTCPP provided Dev with an additional ability. In the following experiments, $15 \mu \mathrm{mol} / \mathrm{g}$ of $\mathrm{Cu}-\mathrm{OBTCPP}_{\mathrm{D}}$ by which every $\mathrm{PAH}$ was retained most strongly was used as the stationary phase of a test column. Figure 5 shows the chromatograms obtained by subjecting, as a sample, benzene, naphthalene, anthracene, phenanthrene, pyrene or triphenylene to $\mathrm{Cu}-\mathrm{OBTCPP}_{\mathrm{D}}(15 \mu \mathrm{mol} / \mathrm{g})$ column in a polar or nonpolar eluent. As can be seen from Fig. 5, PAHs were well retained on a $\mathrm{Cu}-\mathrm{OBTCPP}_{\mathrm{D}}$ column in either a polar or nonpolar eluent, but the theoretical plate number $(N)$ was small in every peak. This can be attributed to the amino groups present on Dev. On the assumption that $\mathrm{Cu}-\mathrm{OBTCPP}_{\mathrm{D}}$ column is a potential preparative column for PAHs, we examined the retention property of said $\mathrm{Cu}-\mathrm{OBTCPP}_{\mathrm{D}}$ column in the following comparative experiments.

Comparison with PYE Column PYE column has a pyrenylethyl group and is well known to exhibit $\pi-\pi$ electron interaction based on hydration energy with a substance having $\pi$ electron in a polar eluent. Accordingly, comparison of retention behavior for simple PAHs between $\mathrm{Cu}-$ $\mathrm{OBTCPP}_{\mathrm{D}}$ and PYE columns may clarify whether or not $\mathrm{Cu}-$ OBTCPP $_{\mathrm{D}}$ has an ability to exhibit interactions such as $\pi-\pi$ electron interaction. We prepared a correlation chart from $k$ values of respective columns obtained using simple PAHs lacking a substituent including benzene, naphthalene, anthracene, phenanthrene, pyrene and triphenylene on respective columns. The results are shown in Figs. 6 and 7. As can be seen from Fig. 6, $k$ values of PAHs on the PYE column elevated as the ring number of PAHs increased. This can be explained by that the hydration energy increases in parallel with the ring number of PAHs. The $\mathrm{Cu}-\mathrm{OBTCPP}_{\mathrm{D}}$ column seems to be in linear correlation with PYE column as far as $k$ values of benzene, naphthalene, phenanthrene and pyrene are

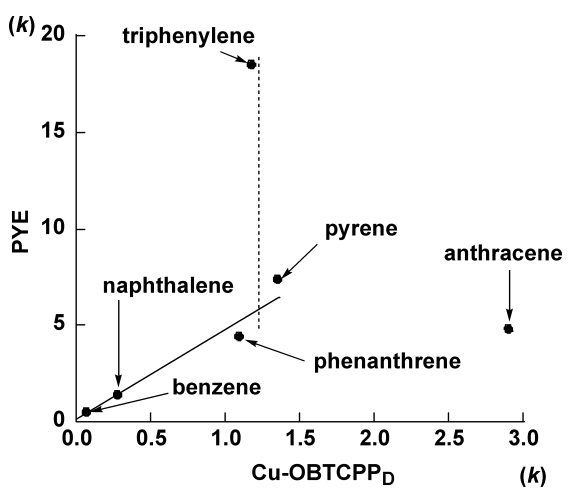

Fig. 6. Correlation of Retention Factor $(k)$ on $\mathrm{Cu}-\mathrm{OBTCPP}_{\mathrm{D}}$ and PYE Columns in $80 \%$ Methanol

concerned. However, no linear correlation is observed regarding $k$ values for anthracene and triphenylene. In addition, the $k$ values on the $\mathrm{Cu}-\mathrm{OBTCPP}_{\mathrm{D}}$ column are generally lower than those on the PYE column. These differences show that $\mathrm{Cu}-\mathrm{OBTCPP}_{\mathrm{D}}$ can recognize $\pi$ electron spreading to a certain limited extent but not $\pi$ electron spreading broadly, which means that the deprivation of planar structure of porphyrin ring by bromination decreased the ability to recognize $\pi$ electron spread. Detailed investigations revealed that, as shown by dashed line in Fig. $6, k$ values on the $\mathrm{Cu}$ OBTCPP $_{D}$ column are almost the same among phenanthrene, pyrene and triphenylene, which comprise benzene rings compactly connected and have almost the same maximum molecular size. These results strongly suggest that $\mathrm{Cu}-\mathrm{OBTCPP}_{\mathrm{D}}$ interacts with whole or part of benzene rings of respective PAHs as shown by shading in Fig. 2. Specifically, the $k$ value is larger for anthracene than pyrene or the like. This is because, in contrast with pyrene or anthracene is composed of benzene rings connected in tandem and has larger maximum molecular size, and furthermore interacts with $\mathrm{Cu}$-OBTCPP through the whole molecule.

In addition, although $\mathrm{Cu}-\mathrm{OBTCPP}_{\mathrm{D}}$ interacts with PAHs through $\pi-\pi$ electron interaction based on hydration energy like PYE column, said interaction is limited to a certain extent and is not strong enough. Furthermore, only a part of $\mathrm{Cu}-\mathrm{OBTCPP}$ participates into such interaction with a $\mathrm{PAH}$ partially in some cases. On the other hand, in non-polar eluents, a linear correlation was observed between $\mathrm{Cu}$ OBTCPP $_{\mathrm{D}}$ and PYE columns regarding $k$ values of PAHs as shown in Fig. 7. Furthermore, $k$ values on the $\mathrm{Cu}-\mathrm{OBTCPP}_{\mathrm{D}}$ column were almost three times as large as those of the PYE column. Under the condition, the PYE column possibly exhibits $\pi-\pi$ electron interactions in a broad sense (e.g., dispersion forces including Van der Waals' forces ${ }^{24)}$ ) which interactions function effectively even in a hydrophobic solvent. Seemingly, Fig. 7 shows that $\mathrm{Cu}-\mathrm{OBTCPP}_{\mathrm{D}}$ and PYE columns function through the same interactions. However, it is almost impossible to expect strong $\pi-\pi$ interactions between a sample and $\mathrm{Cu}$-porphyrin-ring of $\mathrm{Cu}-\mathrm{OBTCPP}$ considering that $\mathrm{Cu}$-TCPP has a large planar structure but cannot exhibit strong $\pi-\pi$ electron interactions ${ }^{18)}$ and $\mathrm{Cu}$-OBTCPP is deprived of the original planar porphyrin ring ${ }^{22)}$ structure by bromination. Accordingly, the apparent linear correlation can be explained by that, in the case of Cu-OBTCPP, a $\mathrm{Cu}^{2+}$ ion is unable to take the square planner structure because of the non-planar structure of porphyrin ring, which brings 


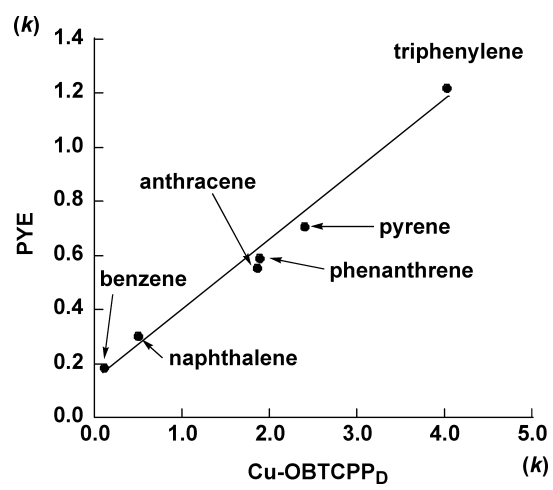

Fig. 7. Correlation of Retention Factor $(k)$ on $\mathrm{Cu}-\mathrm{OBTCPP}_{\mathrm{D}}$ and PYE Columns in $100 \% n$-Hexane

about enhancement of $\pi-d$ interaction wherein $\pi$ electron coordinates to $d$ orbital(s) of a $\mathrm{Cu}^{2+}$ ion. Thus, $\mathrm{Cu}-$ $\mathrm{OBTCPP}_{\mathrm{D}}$ can retain PAHs in a hydrophobic solvent through interactions involving " $\pi$ electron" such as $\pi-\pi$ electronand $\pi-d$-interactions which are different from the " $\pi-\pi$ electron interactions" in a polar solvent. The results also demonstrate that the $\mathrm{Cu}-\mathrm{OBTCPP}_{\mathrm{D}}$ column retains PAHs more strongly than the PYE column. Accordingly, Cu-OBTCPP column is expected to be superior to the PYE column in a non-polar eluent. It is of interesting that the retention property of $\mathrm{Cu}-\mathrm{OBTCPP}_{\mathrm{D}}$ differs from that of the PYE column depending on the polarity of eluent, i.e., polar and nonpolar eluents.

Comparison with PBB Column As a column containing a stationary phase comprising brominated aromatic rings, PBB column, ${ }^{16)}$ is commercially available. To elucidate the effect of bromination on Cu-TCPP, we compared the retention behavior PAHs on a PBB column and that of a $\mathrm{Cu}-$ $\mathrm{OBTCPP}_{\mathrm{D}}$ column. In a polar eluent, the PBB column is reported to interact with halogenated benzene or the like through a weak dispersion force in addition to the $\pi-\pi$ electron interaction. ${ }^{16)}$ We prepared a correlation chart from $k$ values of fluoro-, chloro-, bromo- and iodo-benzenes on $\mathrm{Cu}$ OBTCPP $_{D}$ and PBB columns. The chart is shown in Fig. 8. As can be seen from Fig. 8, the PBB column retained substituted benzenes strongly and unsubstituted benzenes weakly as reported previously. ${ }^{16)}$ In the case of the $\mathrm{Cu}-\mathrm{OBTCPP}_{\mathrm{D}}$ column, $k$ values for halogenated benzenes are almost the same as those for benzenes as shown in Fig. 8. The results seem to suggest the linear correlation between $\mathrm{Cu}-\mathrm{OBTCPP}{ }_{\mathrm{D}}$ and $\mathrm{PBB}$ columns depending on perspective; however, the variation of $k$ values on the $\mathrm{Cu}-\mathrm{OBTCPP}_{\mathrm{D}}$ column are generally smaller. Therefore, the retention behavior of halogenated benzenes on the $\mathrm{Cu}-\mathrm{OBTCPP}_{\mathrm{D}}$ column for is considered to be dominated mainly by weak $\pi-\pi$ electron interactions like unsubstituted benzenes. It is reported that a PBB column has a shape-recognition ability for $\pi$ electron cloud of fullerenes in a nonpolar eluent. ${ }^{16)}$ In this experiment, we used the same halogenated benzenes as those used in the experiments in a polar eluent, because medicines do not contain a compound like fullerenes at resent. The resultant correlation chart is given in Fig. 9. As can be seen from Fig. 9, the PBB column retains chloro- and bromo-benzenes almost equally to benzene, but slightly strongly fluoro- and iodo-benzenes than benzene. The results above suggest that the PBB column hardly recognizes these compounds, in other words, the PBB

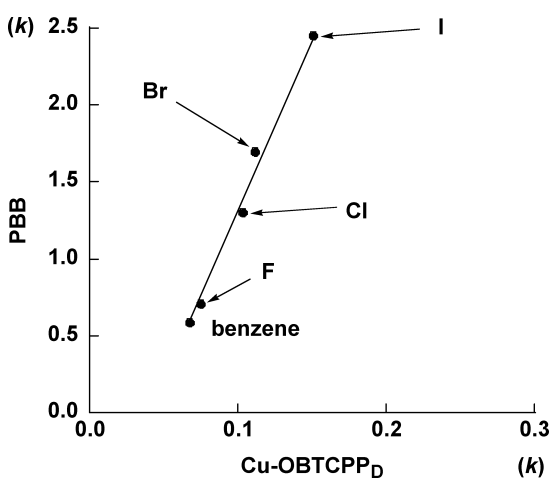

Fig. 8. Correlation of Retention Factor $(k)$ on $\mathrm{Cu}-\mathrm{OBTCPP}_{\mathrm{D}}$ and $\mathrm{PBB}$ Columns in $80 \%$ Methanol

F: fluorobenzene, $\mathrm{Cl}$ : chlorobenzene, $\mathrm{Br}$ : bromobenzene, I: iodobenzene.

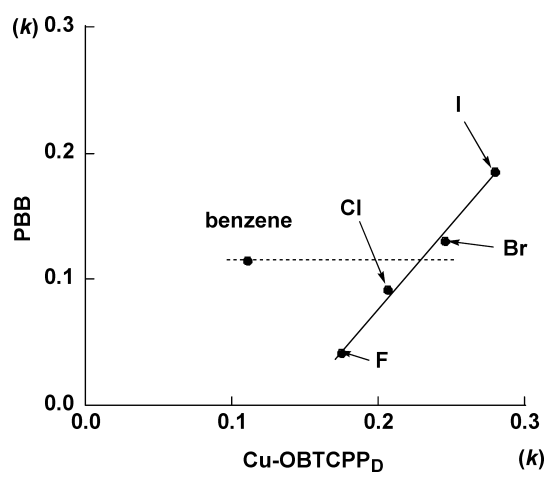

Fig. 9. Correlation of Retention Factor $(k)$ on $\mathrm{Cu}-\mathrm{OBTCPP}_{\mathrm{D}}$ and $\mathrm{PBB}$ Columns in $100 \% n$-Hexane

$\mathrm{F}$ : fluorobenzene, $\mathrm{Cl}$ : chlorobenzene, $\mathrm{Br}$ : bromobenzene, I: iodobenzene.

column does not exhibit marked interaction with these benzene derivatives. On the other hand, the $\mathrm{Cu}-\mathrm{OBTCPP}_{\mathrm{D}}$ column retains halogenated benzenes more strongly than benzene as shown in Fig. 9. In addition, the retention by the $\mathrm{Cu}-$ $\mathrm{OBTCPP}_{\mathrm{D}}$ column increases in accordance with the molecular size of halogenated benzenes. The fact that the $\mathrm{Cu}-$ $\mathrm{OBTCPP}_{\mathrm{D}}$ column retains strongly halogenated benzenes which are more polarized than benzene suggests the possible contribution of electrostatic force even a little.

Comparison with NPE Column As described above, the $\mathrm{Cu}-\mathrm{OBTCPP}_{\mathrm{D}}$ column possibly interacts with a sample compound through electrostatic interactions. In the present experiment, an NPE column ${ }^{17}$ ) exhibiting dipole-dipole interactions, which are one of electrostatic interactions, with a sample compound having a dipole in a nonpolar eluent was used. The NPE column has nitrophenyl group and therefore is thought to exhibit interaction mainly dominated by $\pi-\pi$ electron interaction in a polar eluent, and electrostatic interaction in a non-polar eluent. We therefore compared the retention property of NPE column and that of the $\mathrm{Cu}$ $\mathrm{OBTCPP}_{\mathrm{D}}$ column using nitrobenzene and benzonitrile having a dipole.

Table 1 shows $k$ values of nitrobenzene and benzonitrile on the $\mathrm{Cu}-\mathrm{OBTCPP}_{\mathrm{D}}$ and NPE columns in various eluents. As can be seen from Table 1, the NPE column retained these samples more strongly in a polar eluent, wherein the $\pi-\pi$ electron interaction is dominant. In a nonpolar eluent, both of $\mathrm{Cu}-\mathrm{OBTCPP}_{\mathrm{D}}$ and NPE columns retained the sample compounds strongly. This indicates that the $\mathrm{Cu}-\mathrm{OBTCPP}_{\mathrm{D}}$ col- 
Table 1. Correlation of Retention Factors ( $k$ Values) of Nitrobenzene and Benzonitrile on the $\mathrm{Cu}-\mathrm{OBTCPP}_{\mathrm{D}}$ and NPE Columns

\begin{tabular}{cccccc}
\hline \hline & \multicolumn{2}{c}{$100 \% n$-hexane } & & \multicolumn{2}{c}{$80 \%$ methanol } \\
\cline { 2 - 3 } \cline { 5 - 6 } & Nitrobenzene & Benzonitrile & & Nitrobenzene & Benzonitrile \\
\hline Cu-OBTCPP & & & & \\
NPE column & 3.16 & 8.25 & & 0.11 & 0.11 \\
& 1.21 & 8.57 & & 0.29 & 0.54 \\
\hline
\end{tabular}

umn, similar to the NPE column, has an ability to exhibit dipole-dipole interaction, one of electrostatic interactions in non-polar eluent, whose ability should be one of characteristics of a Cu-OBTCPP ${ }_{\mathrm{D}}$ column undergone bromination.

\section{Conclusion}

$\mathrm{Cu}-\mathrm{OBTCPP}_{\mathrm{D}}$ was revealed to be a novel prospective HPLC stationary phase capable of functioning through specific separation mechanisms. For example, $\mathrm{Cu}-\mathrm{OBTCPP}_{\mathrm{D}}$ can exhibit strong interactions involving $\pi$ electron or electrostatic interaction in a non-polar eluent. Although continuous researches for optimization, improvement of performance, and the like, would be our future task, OBTCPP ${ }_{D}$ is a promising stationary phase useful in the analysis of medicines or starting materials therefor having $\pi$ electron.

\footnotetext{
References

1) Sun X., Yang X., Wang E., Anal. Chim. Acta, 547, 153-157 (2005).

2) Panteghini M., Forest J. C., Clin. Chim. Acta, 355, 1-12 (2005).

3) Tasao R., Deng Z., J. Chromatogr. B, 812, 85-99 (2004).

4) Hewitt L. M., Marvin C. H., Mutat. Res., 589, 208-232 (2005).

5) Moret S., Conte L. S., J. Chromatogr. A, 882, 245-253 (2000).

6) Jaouen-Madoulet A., Abarnou A., Le Guellec A.-M., J. Chromatogr.
}

$A, \mathbf{8 8 6}, 153-173$ (2000).

7) Filipkowska A., Lubecki L., Kowalewska C., Anal. Chim. Acta, 547, $243-254$ (2005)

8) Lage Yusty M. A., Cortizo Davina J. L., Food Control, 16, 59-64 (2005).

9) Delgado B., Pino V., Ayala J. H., Gonzalez V., Afonso A. M., Anal. Chim. Acta, 518, 165-172 (2004).

10) Jinno K., Nagoshi T., Tanaka N., Okamoto M., Fetzer J. C., Biggs W. R., J. Chromatogr., 386, 123-135 (1987).

11) Kannan N., Petrick G., Brihn R., Schulz-Bull D. E., Chemosphere, 37, 2385-2393 (1998).

12) Mifune M., Odo J., Otsuki Y., Mitsuhashi M., Mori Y., Gassim A. E. H., Haginaka J., Saito Y., Anal. Sci., 7, 805-806 (1991).

13) Kibbey C. E., Meyerhoff M. E., J. Chromatogr. A, 641, 49-55 (1993).

14) Chen S., Meyerhoff M. E., Anal. Chem., 70, 2523-2524 (1998).

15) Mifune M., Mori Y., Onoda M., Iwado A., Motohashi N., Haginaka J., Saito Y., Anal. Sci., 14, 1127-1131 (1991).

16) Kimata K., Hirose T., Moriuchi K., Hosoya K., Araki T., Tanaka N., Anal. Chem., 67, 2556-2561 (1995).

17) Kimata K., Hosoya K., Yanaka N., Araki T., Patteson D. G. Jr., J. Chromatogr. A, 595, 77-88 (1992).

18) Mifune M., Minato K., Kitamura Y., Okazaki K., Iwado A., Akizawa H., Haginaka J., Motohashi N., Saito Y., Talanta, 63, 1035-1038 (2004).

19) Pasternack R. F., Francesconi L., Raff D., Spiro E., Inorg. Chem., 12, 2606-2611 (1973).

20) Tabata M., Nishimoto J., Ogata A., Kusano T., Nahar N., Bull. Chem. Soc. Jpn., 69, 673-677 (1996).

21) Iwado A., Mifune M., Harada R., Oda J., Motohashi N., Saito Y., Chem. Pharm. Bull., 47, 1301-1304 (1999).

22) Barinic-Haberle I., Liochev S. I., Spasojevic I., Fridovich I., Arch. Biochem. Biophys., 343, 225-233 (1997).

23) Gruden-Pavlovic M., Grubisic S., Niketic S. R., J. Inorg. Biochem., 98, 1293 - 1302 (2004).

24) Turowski M., Morimoto T., Kimata K., Monde H., Ikegami T., Hosoya K., Tanaka N., J. Chromatogr. A, 911, 177-190 (2001). 